\title{
石油沥青基纳米碳球的制备及其电化学性能研究
}

\author{
潘海玲 $^{a, b}$ 李丽 ${ }^{b} \quad$ 刘江华 ${ }^{c}$ 贾殿赠 $*^{a}, a$ \\ ( ${ }^{a}$ 新疆大学应用化学研究所 清洁能源材料与技术教育部重点实验室 先进功能材料自治区级重点实验室 \\ 乌鲁木齐 830046) \\ ( ${ }^{b}$ 新疆大学化学化工学院 乌鲁木齐 830046) \\ ( ${ }^{c}$ 新疆油田公司工程技术研究院 克拉玛依 834000)
}

\begin{abstract}
摘要 以石油沥青为碳源, 在空气中将其加热到 $450{ }^{\circ} \mathrm{C}$ 制备出纳米碳球 $(\mathrm{CNBs})$, 经 $1500{ }^{\circ} \mathrm{C}$ 氮气气氛中加热纳米碳球 3 $\mathrm{h}$ 后得到高温处理的纳米碳球(H-CNBs). 采用扫描电镜、透射电镜、X 射线衍射、拉曼光谱和红外光谱对所制备的产 物进行了结构表征. 结果表明: 制得的 CNBs 的粒径在 50 80 nm 之间, 石墨化程度不高, H-CNBs 粒径没有改变但石 墨化程度有所提高, 推测了 CNBs 的形成机理. 用恒流充放电测试分别对 CNBs 和 H-CNBs 的电化学性能进行了研究, 在电流密度为 $1 \mathrm{C}$ 时, 其首次放电比容量和经过 100 圈之后的放电比容量分别为 $1260 \mathrm{mAh} / \mathrm{g}$ 和 $500 \mathrm{mAh} / \mathrm{g}, 413 \mathrm{mAh} / \mathrm{g}$ 和 $200 \mathrm{mAh} / \mathrm{g}$ 之上, 同时这两种纳米碳球的首次充放电的库伦效率较低, 分别经过 10 圈和 30 圈后可以稳定在 $98 \%$ 左 右. CNBs 在经历 $0.1 \mathrm{C}, 1 \mathrm{C}, 5 \mathrm{C}, 10 \mathrm{C}$ 循环回到 $0.1 \mathrm{C}$ 时, 容量几乎完全恢复.
\end{abstract}

关键词 石油沥青; 纳米碳球; 电极材料; 比容量

\section{Preparation and Electrochemical Performance of Carbon Nanoballs from Petroleum Asphalt}
Pan, Hailing ${ }^{a, b}$
$\mathrm{Li}, \mathrm{Li}^{b}$
Liu, Jianghua ${ }^{c}$
Jia, Dianzeng*,a

$\left({ }^{a}\right.$ Key Laboratory of Material and Technology for Clean Energy, Ministry of Education, Key Laboratory of Advanced Functional Materials, Autonomous Region, Institute of Applied Chemistry, Xinjiang University, Urumqi 830046)

$\left({ }^{b}\right.$ School of Chemistry and Chemical Engineering, Xinjiang University, Urumqi 830046)

( ${ }^{c}$ Engineering Technology Research Institute of Xinjiang Oilfield Company, Karamay 834000)

\begin{abstract}
In this work, a simple low-temperature method has been employed to synthesize carbon nanoballs (CNBs). In a typical procedure, $1 \mathrm{~g}$ of petroleum asphalt in an alumina boat was directly heated to $450{ }^{\circ} \mathrm{C}$ at a rate of $10{ }^{\circ} \mathrm{C} / \mathrm{min}$ in air in a conventional box furnace to obtain the CNBs. Subsequently, the synthesized CNBs were treated in tubular furnace at $1500{ }^{\circ} \mathrm{C}$ under nitrogen atmosphere for $3 \mathrm{~h}$ and the obtained products were denoted as H-CNBs. The as-synthesized CNBs and H-CNBs were characterized by scanning electron microscopy, transmission electron microscopy, X-ray diffraction, Raman spectroscopy, and infrared spectroscopy. It was found that the diameters of CNBs and H-CNBs were in the range of $50 \sim 80$ $\mathrm{nm}$, and both of them showed a relatively low degree of graphitization. Compared with the CNBs, the H-CNBs showed a slightly higher degree of graphitization than the CNBs. A proposed formation mechanism of the CNBs is suggested to be as follows. Firstly, petroleum asphalt decomposed and formed a large amount of hydrocarbons at $220 \sim 500{ }^{\circ} \mathrm{C}$, such as benzene, methane, acetylene and so on. Carbon atoms from thermal cracking products of hydrocarbons deposited on the substrate in the absence of $\mathrm{O}_{2}$. Carbon atoms developed to CNBs as the temperature increased and the reaction time prolonged. The $\mathrm{CNBs}$ and $\mathrm{H}-\mathrm{CNBs}$ were used as anode material in lithium ion batteries. The electrochemical performance was studied by galvanostatic charge/discharge at ambient temperature. The CNBs as anode materials exhibited a high initial capacity of 1260 $\mathrm{mAh} / \mathrm{g}$ at a current of $1 \mathrm{C}$, which stabilized at around $500 \mathrm{mAh} / \mathrm{g}$ after 100 cycles. In contrast, the H-CNBs showed an initial capacity of $413 \mathrm{mAh} / \mathrm{g}$ under the same electrochemical conditions, which stabilized at $200 \mathrm{mAh} / \mathrm{g}$ after about 20 cycles with slight increase upon further cycling. Coulombic efficiency of as high as $98 \%$ was recorded after initial cycling for 10 cycles and 30 cycles for CNBs and $\mathrm{H}-\mathrm{CNBs}$, respectively. After cycling at different currents from $0.1 \mathrm{C}$, to $1 \mathrm{C}, 5 \mathrm{C}$, and $10 \mathrm{C}$, the capacity of CNBs was almost fully recovered to the initial capacity at $0.1 \mathrm{C}$.

Keywords petroleum asphalt; carbon nanoballs; electrode material; specific capacity
\end{abstract}

\footnotetext{
*E-mail: dzj@xju.edu.cn

Received November 30, 2012; published March 21, 2013.

Project supported by the Xinjiang Autonomous Region of high-tech projects (No. 201016118) and the Xinjiang Autonomous Region of major projects: (No. 201130113-1).

项目受新疆自治区高技术项目(No. 201016118)和新疆自治区重大专项(No. 201130113-1)资助.
} 


\section{1 引言}

自从 1991 年日本科学家 Iijima 发现了碳纳米管 ${ }^{[1]}$, 至今纳米碳材料已得到了飞速发展. 纳米碳球(Carbon Nanoballs, 简称 CNBs) 作为纳米碳材料成员之一, 由于 其优良的物理化学性能, 被广泛作为电极材料 ${ }^{[2 \sim 4]}$ 、药 物载体 ${ }^{[5]}$ 、催化剂载体 ${ }^{[6,7]}$ 和储能材料 ${ }^{[8,9]}$. 用于制备纳米 碳球材料的碳源种类繁多, 从碳源的存在状态讲, 主要 分为气态碳源 ${ }^{[10 ~ 12]}$ 、液态碳源 ${ }^{[13]}$ 和固态碳源 ${ }^{[14,15]}$. 其中 气态碳源是发展最早最成熟的, 经常被研究的有甲 烷 ${ }^{[16]}$ 、乙炔 ${ }^{[17]}$ 、乙烯 ${ }^{[18]}$ 等, 但是由于它的成本较高, 储 存不便, 在用于制备纳米碳材料方面较固态碳源有一定 的劣势. 固态碳源是近几年刚刚发展起来的, 主要包括 沥青和煤, 由于其原料易得, 成本低廉且富含碳近年来 得到国内部分院校的关注 ${ }^{[19,20]}$. 如何把廉价碳源经过一 个操作简单、低耗能的过程, 转化为功能化、高附加值 的窄粒径分布的纳米碳球是一个值得研究的课题.

太原理工大学许并社等 ${ }^{[21,22]}$ 分别以脱油沥青和煤 焦油沥青为源, 在 $\mathrm{Ar}$ 气保护下升温至 $900 \sim 1100{ }^{\circ} \mathrm{C}$, 制 备出粒径在 $100 \sim 1000 \mathrm{~nm}$ 之间的纳米碳球. 北京化工 大学沈曾民等 ${ }^{[23]}$ 以石油渣油沥青为碳源, 在温度为 320 ${ }^{\circ} \mathrm{C}$ 时, 采用乳液法制备出了粒径为数十微米的中间相碳 微球. 到目前为止, 有关无催化剂下低温加热石油沥青 制备小粒径 CNBs 及其电化学性质尚未见报道.

本文以新疆石油产品的附属品石油沥青为原料, 在 $450{ }^{\circ} \mathrm{C}$ 无催化剂的条件下制备出粒径在 50 $80 \mathrm{~nm}$ 之间 的 CNBs. 该方法与上述方法不同的是, 它不需要催化 剂, 在较低的温度下制备出小粒径的 CNBs, 并提出了 可能的生长机理. 研究了 CNBs 和其在氮气气氛中高温 处理后的 H-CNBs 的电化学性能.

\section{2 结果与讨论}

\section{1 石油沥青的热分析}

让石油沥青在空气中以 $10{ }^{\circ} \mathrm{C} / \mathrm{min}$ 的升温速率由室 温升至 $1100{ }^{\circ} \mathrm{C}$ 进行热解, 所得热解热重分析曲线(TG) 如图 1 所示. 从图 1 可以看出, 石油沥青热解过程大致 可以分为三个阶段. 从室温到 $220{ }^{\circ} \mathrm{C}$ 为第一阶段, 主要 是干燥脱气过程, 失重曲线基本处于水平状态, 失重不 明显. 第二阶段为 $220 \sim 380{ }^{\circ} \mathrm{C}$, 石油沥青在此温度段 发生热解，此阶段主要发生石油沥青的一些弱键的断裂， 其中包括外围官能团的脱落和杂原子键的断裂, 同时伴 有低分子烃类如甲烷、乙炔等的物理挥发; 第三阶段为 $380 \sim 500{ }^{\circ} \mathrm{C}$, 随着炉体加热, 温度进一步升高后, 热失 重曲线陡然下降, 石油沥青开始激烈分解, 同时氧气参 与反应过程, 发生断链以及裂环反应, 强键遭到破坏, 使得大分子分裂为小的分子, 变为气态挥发分, 最后剩 余无机盐类残余, 完成石油沥青从固相升温到液相, 再 到气相和固相的转变.

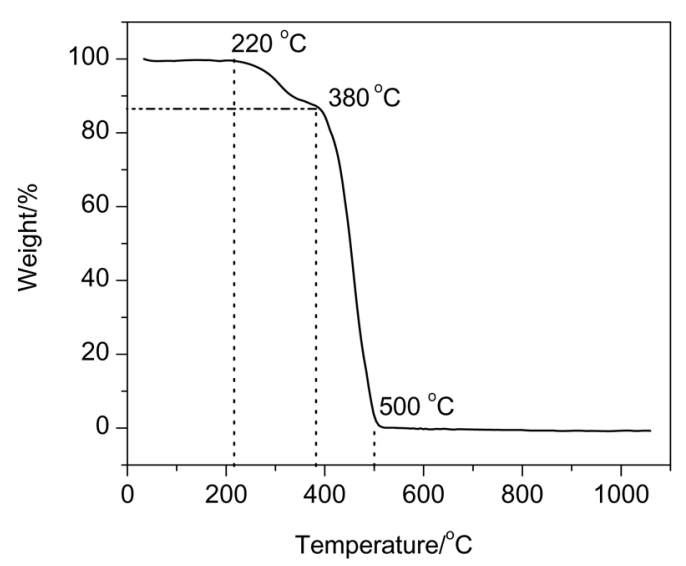

图 1 石油沥青在空气氛围中的 TG 分析曲线

Figure 1 TG curves for petroleum asphalt in air atmosphere

\section{2 纳米碳球的 SEM 和 TEM 分析}

对石油沥青为碳源加热到 $450{ }^{\circ} \mathrm{C}$ 所得产物进行了 SEM 分析, 结果见图 2. 其中图 2a 为石英基底收集的样 品 SEM 照片, 从图中可以看出, 在石英基底的表面有大 面积的准球状颗粒, 球状颗粒的尺寸分布均匀, 直径小 于 $100 \mathrm{~nm}$. 此外, 我们发现准球状颗粒之间出现少量黏 连现象, 说明准球状颗粒的表面上可能有少量没有完全 分解的焦油. 为了进一步确定收集样品的基底对产物的 形貌有无影响，我们分别采用刚玉和铁作为基底收集产 物, 对所得样品进行了 SEM 分析, 结果如图 $2 b$ 和图 $2 \mathrm{c}$ 所示. 从图中可以看出样品均为类球状颗粒且粒径分布 均匀, 直径小于 $100 \mathrm{~nm}$, 以上结果说明样品的形貌与基 底无关. 为了进一步确定类球状颗粒的微结构, 将类球 状颗粒在无水乙醇中超声 $20 \mathrm{~min}$ 后进行了 TEM 分析, 结果见图 $2 \mathrm{~d}$. 从图可以看出产物为球形颗粒, 粒径在 $50 \sim 80 \mathrm{~nm}$ 之间, 这与 SEM 分析结果一致. 将 $450{ }^{\circ} \mathrm{C}$ 所 得产物在氮气气氛中以 $10{ }^{\circ} \mathrm{C} / \mathrm{min}$ 的加热速率加热到 $1500{ }^{\circ} \mathrm{C}$ 并保温 $3 \mathrm{~h}$, 对所制备的产物进行了 TEM 分析, 结果见图 2e. 从图可以看出, 加热后的产物为球形颗粒, 粒径在 50 80 nm 之间, 另外纳米碳球发生了少许的收 缩变形, 可能是由于纳米碳球表面及内部碳原子高温重 排造成碳球整体收缩所致.

\section{3 纳米碳球的 XRD 分析}

对上述 $450{ }^{\circ} \mathrm{C}$ 和 $1500{ }^{\circ} \mathrm{C}$ 所得产物进行了 XRD 分析, 结果见图 3. 图 $3 \mathrm{a}$ 和 $3 \mathrm{~b}$ 分别为 $450{ }^{\circ} \mathrm{C}$ 和 $1500{ }^{\circ} \mathrm{C}$ 所得产 物的 XRD 图谱. 图中 $2 \theta$ 为 $25.5^{\circ}, 42.5^{\circ}$ 和 $25.5^{\circ}, 43.1^{\circ}$ 的 衍射峰分别与碳的(002)和(100)面的衍射峰位相对应, 此外没有其余的杂质峰，表明在石英基底表面生长的球 形颗粒均为碳球. 我们发现 $450{ }^{\circ} \mathrm{C}$ 和 $1500{ }^{\circ} \mathrm{C}$ 所得产物 的(002)晶面对应的衍射峰 $\left(2 \theta=25.5^{\circ}\right)$ 比完美结晶度的 纯相石墨 $(002)$ 晶面对应的衍射峰 $\left(2 \theta\right.$ 为 $\left.26.5^{\circ}\right)$ 小, 这说 明所制备样品的碳原子堆积有序化程度较低. 另外, 450 ${ }^{\circ} \mathrm{C}$ 所得产物(CNBs) 的(002) 晶面对应的衍射峰 $(2 \theta=$ 


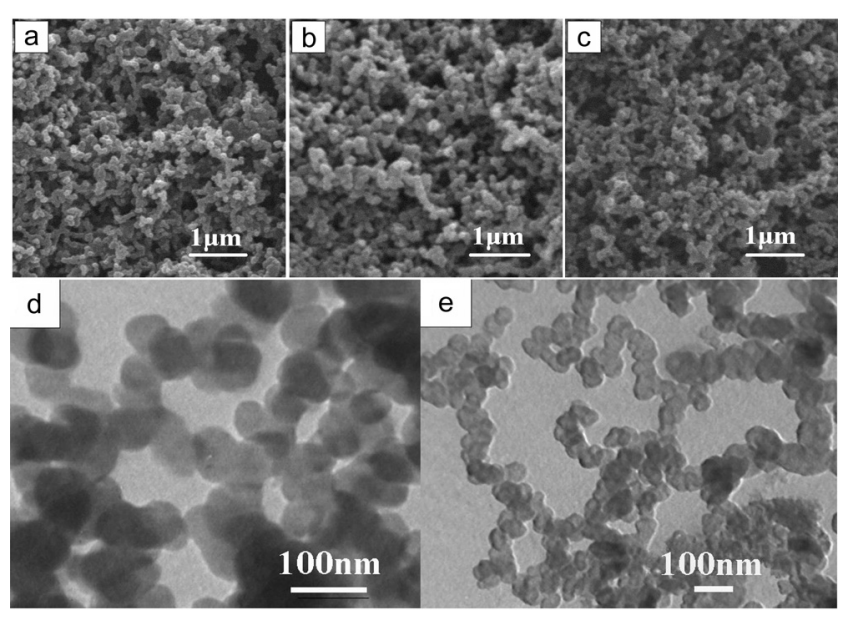

图 2 CNBs 的 SEM 图(a) @石英基底, (b) @ 刚玉基底, (c) @铁基底及 CNBs (d) 和 H-CNBs (e)的 TEM 图

Figure 2 SEM images of CNBs (a) @quartz tablet, (b) @ corundum plate, (c)@iron base, and TEM images of CNBs (d) and H-CNBs (e)

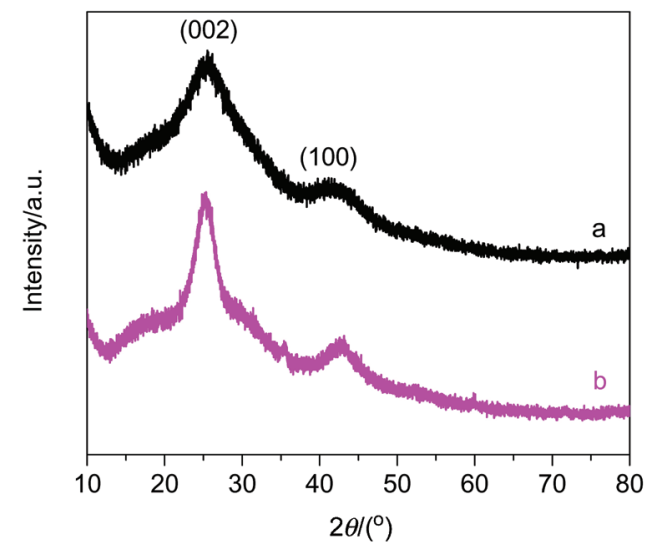

图 3 CNBs (a) 和 $\mathrm{H}-\mathrm{CNBs}(\mathrm{b})$ 的 XRD 图谱

Figure 3 XRD patterns of CNBs (a) and H-CNBs (b)

$25.5^{\circ}$ )的半峰宽较大, 通过 Bragg 方程计算了所得 CNBs 的层间距 $d_{002}$ 为 $0.3488 \mathrm{~nm}$, 比结构完美的石墨晶体的 层间距 $0.3354 \mathrm{~nm}$ 大, 这也说明所得 CNBs 的石墨化程 度不高. 与 $\mathrm{CNBs}$ 相比, $1500{ }^{\circ} \mathrm{C}$ 所得产物( $\left.\mathrm{H}-\mathrm{CNBs}\right)$ 在 $2 \theta=25.5^{\circ}$ 处的峰宽相对变小, 同时(100)面的衍射峰位 向左发生了偏移, 表明 H-CNBs 的结晶度与 CNBs 相比 相对增高.

\section{4 纳米碳球的 Raman 分析}

为了进一步确定所得纳米碳球的石墨化程度, 对 CNBs 和 H-CNBs 进行了 Raman 分析, 结果如图 4 所示. 从图中观测到波数为 1348 和 $1586 \mathrm{~cm}^{-1}$ 的两个拉曼峰. 1348 和 $1586 \mathrm{~cm}^{-1}$ 两个强峰分别为 $\mathrm{D}$ 峰和 $\mathrm{G}$ 峰, 它们为 碳材料的特征峰. 其中 $\mathrm{D}$ 峰主要是由于碳材料缺陷引起, $\mathrm{G}$ 峰与碳材料的 $\mathrm{C}-\mathrm{C}$ 键 $\mathrm{sp}^{2}$ 杂化振动相关. 通常用 $\mathrm{G}$ 峰和 $\mathrm{D}$ 峰的相对强度比值 $\left(I_{\mathrm{G}} / I_{\mathrm{D}}\right)$ 来描述碳材料结构中点 缺陷的密集度, $I_{\mathrm{G}} / I_{\mathrm{D}}$ 值越大, 缺陷度越小. 本实验制备 的 CNBs 和 $\mathrm{H}-\mathrm{CNBs}$ 的 $I_{\mathrm{G}} / I_{\mathrm{D}}$ 值分别为 1.2 和 1.3 , 石墨的 $I_{\mathrm{G}} / I_{\mathrm{D}}$ 为 $5.172^{[24]}$, 说明样品 $\mathrm{CNBs}$ 和 $\mathrm{H}-\mathrm{CNBs}$ 的缺陷比
石墨多, 其石墨化程度不高. 另外, H-CNBs 的 $I_{\mathrm{G}} / I_{\mathrm{D}}$ 值比 $\mathrm{CNBs}$ 的高, 说明 H-CNBs 的缺陷减少, 这与 XRD 分析 结果相一致.

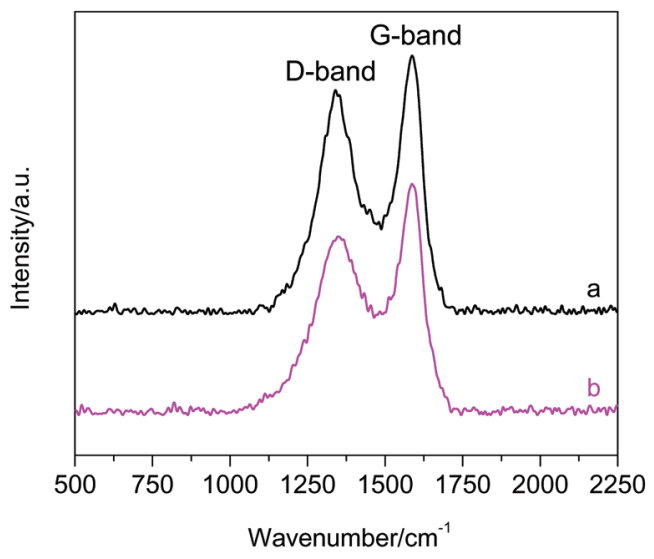

图 $4 \mathrm{CNBs}$ (a)和 H-CNBs (b)的拉曼光谱图

Figure 4 Raman spectra of CNBs (a) and H-CNBs (b)

\section{5 纳米碳球的 IR 分析}

为了进一步研究两种纳米碳球的表面结构, 对所制 备的 CNBs 和 H-CNBs 进行了 IR 分析, 结果见图 5. 图 $5 \mathrm{a}$ 和 $5 \mathrm{~b}$ 分别为 CNBs 和 $\mathrm{H}-\mathrm{CNBs}$ 的 IR 图谱. 图 $5 \mathrm{a}$ 中 $3444 \mathrm{~cm}^{-1}$ 为样品 $\mathrm{CNBs}$ 中水的 $\mathrm{OH}$ 伸缩振动峰, 1384 $\mathrm{cm}^{-1}$ 处的吸收峰为水的 $\mathrm{OH}$ 的弯曲振动峰; 1731,1267 $\mathrm{cm}^{-1}$ 的吸收峰为 $\mathrm{C}=\mathrm{O}$ 的伸缩振动峰; $1553,668 \mathrm{~cm}^{-1}$ 处的吸收峰分别为苯环中双键特征振动吸收峰和苯环 的 $\mathrm{C}-\mathrm{H}$ 的弯曲振动吸收峰; 另外在 $2354 \mathrm{~cm}^{-1}$ 处出现 了一个较宽的吸收带是羧酸分子内羰基和羟基的强烈 缔合形成的. 以上结果表明所制备的 CNBs 的表面存在 水分子、羧基、羟基、羰基和取代的苯环等多种基团. 我 们发现, 与 CNBs 相比, H-CNBs 在 $1731 \mathrm{~cm}^{-1}$ 处的 $\mathrm{C}=\mathrm{O}$ 吸收峰明显减弱, 位于 $1267 \mathrm{~cm}^{-1}$ 处的 $\mathrm{C}=\mathrm{O}$ 吸收峰几 乎完全消失, 而位于 3444 和 $1384 \mathrm{~cm}^{-1}$ 处的 $\mathrm{OH}$ 吸收峰 却明显增强, 主要是由于 $\mathrm{CNBs}$ 在高温环境下, 碳球上 的 $\mathrm{C}=\mathrm{O}$ 与碳球上的氢原子或者官能团发生反应, 转化 成为 $\mathrm{OH}$ 官能团.

\section{6 纳米碳球的形成机理}

关于在空气中纳米碳球的生长主要有一种广泛被 接受的理论，即热解条件下均相成核的“液滴理论”。该 理论认为反应物在一定的温度下发生热解反应生成较 高分子量的烃类物质，当这些烃类物质的蒸气压超过其 饱和蒸气压时, 发生凝聚, 生成微小液滴. 液滴继续热 解发生脱氢反应而碳化，形成纳米碳晶，随着反应时间 的延长, 形成了纳米碳球. 在本实验中以石油沥青为源 在空气中加热制备出纳米碳球，基于上述 “液滴理论”, 我们提出了 $\mathrm{CNBs}$ 的生长机理. 石油沥青在 $220 \sim 500{ }^{\circ} \mathrm{C}$ 发生热解反应(如图 1 所示), 产生大量烃类化合物, 烃 类化合物因缺氧发生脱氢而炭化沉积在基底上，随着时 


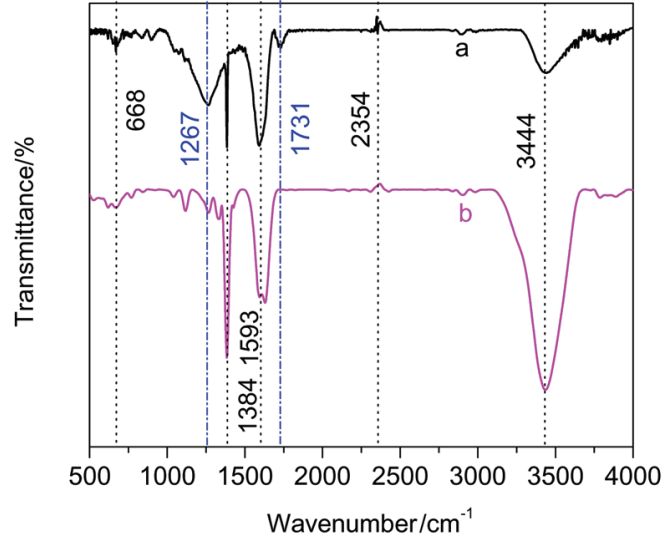

图 $5 \mathrm{CNBs}(\mathrm{a})$ 和 H-CNBs (b)的红外谱图

Figure 5 IR spectra of CNBs (a) and $\mathrm{H}-\mathrm{CNBs}$ (b)

间的延长和温度的升高, 在基底上生长出纳米碳球, 其 生长过程示意图如图 6 所示. 首先, 石油沥青受热分解 生成焦油、低碳烃类和少量的灰分, 其中, 一部分焦油 和低碳烃类化合物在缺氧条件下热解脱氢、碳化形成碳 核, 同时少量焦油和低碳烃类化合物在空气中完全燃烧 生成一氧化碳、二氧化碳和水, 并放出大量的热, 使反 应部分区域的温度迅速升高, 导致了未参与反应的焦油 分解形成烃等有机物, 这些有机物吸附在碳核的表面为 碳球生长提供了物质基础, 随着反应时间的延长, 基底 上的碳核逐渐长大生长出纳米碳球. 此外, 由于在碳球 表面上还有少量未分解的焦油, 这些焦油使纳米碳球之 间发生了粘连, 如图 $2 \mathrm{~d}$ 所示. 因此我们认为在石油沥青 热解的早期阶段, 重焦油分子和少量烃类有机物热解脱 氢是形成 CNBs 晶核的主要来源, 之后, 来自一氧化碳、 甲烷和氢气等轻质气体与焦油的缺氧反应促使 $\mathrm{CNBs}$ 的 生长.

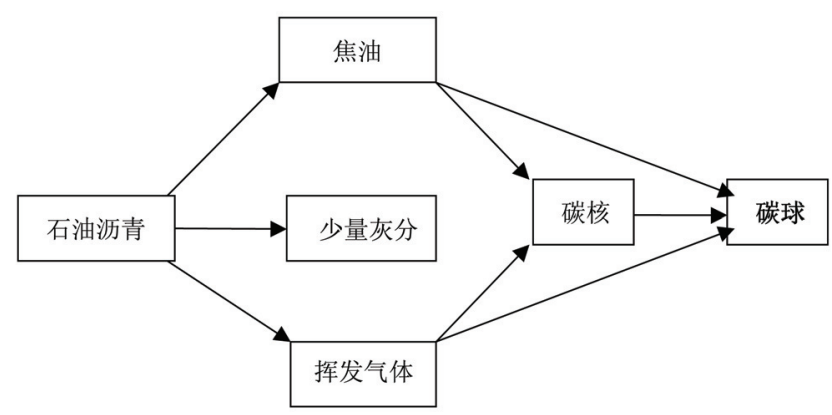

图 6 空气中石油沥青加热制备 $\mathrm{CNBs}$ 的过程示意图

Figure 6 Scheme of CNBs formation process by heating petroleum asphalt in air

\section{7 纳米碳球的电化学性质}

目前人们对气态碳源制备的 CNBs 的电化学性质进 行了大量的研究, 然而鲜见有关石油沥青制备所得 $\mathrm{CNBs}$ 的电化学性质的报道. 本实验分别以 CNBs 和 $\mathrm{H}-\mathrm{CNBs}$ 作为负极材料在 $1 \mathrm{C}(372 \mathrm{mAh} / \mathrm{g})$ 下进行了循环
性能研究，结果如图 7 所示. 从图 7a 中分析可得，试样 $\mathrm{CNBs}$ 首次放电量可达 $1260 \mathrm{mAh} / \mathrm{g}$ ，从第 2 圈开始 $\mathrm{CNBs}$ 负极材料放电容量开始有不同程度的衰减. 循环 50 圈 之后，充放电曲线几乎重合，其循环稳定性变得较好， 从 50 100 圈放电容量稳定在 $500 \mathrm{mAh} / \mathrm{g}$ 之上, 较纯相 石墨的理论放电比容量 $(372 \mathrm{mAh} / \mathrm{g})$ 要高. 同时其库伦 效率首圈仅为 $63 \%$, 之后便相对稳定，10 圈之后稳定在 $98 \%$ 左右. 从图 $7 \mathrm{~b}$ 中分析可知试样 H-CNBs 首次放电容 量为 $413 \mathrm{mAh} / \mathrm{g}$, 但从第二圈开始就恒定在 $200 \mathrm{mAh} / \mathrm{g}$ 左右，同时有缓慢的上升趋势，其库伦效率首圈仅为 $43 \%, 30$ 圈之后稳定在 $98 \%$ 左右. 目前普遍认为样品的 放电容量与样品的比表面积有关, 表面积越大, 放电容 量就越大 ${ }^{[25]}$. 本实验所制备的 CNBs 的粒径在 $50 \sim 80$ $\mathrm{nm}$ 之间，由于其粒径小，所以比表面积大，使得 CNBs 具有较大的电容量. 此外, 放电容量还与 CNBs 上带有 的官能团和缺陷数量成正比, 从 CNBs 的红外光谱图 5 可知, CNBs 表面存在许多含氧官能团，增加了 CNBs 表 面的活性点，这也使得该样品 CNBs 的放电容量高于纯 相的石墨理论容量. 高温处理后的 H-CNBs 形貌出现收
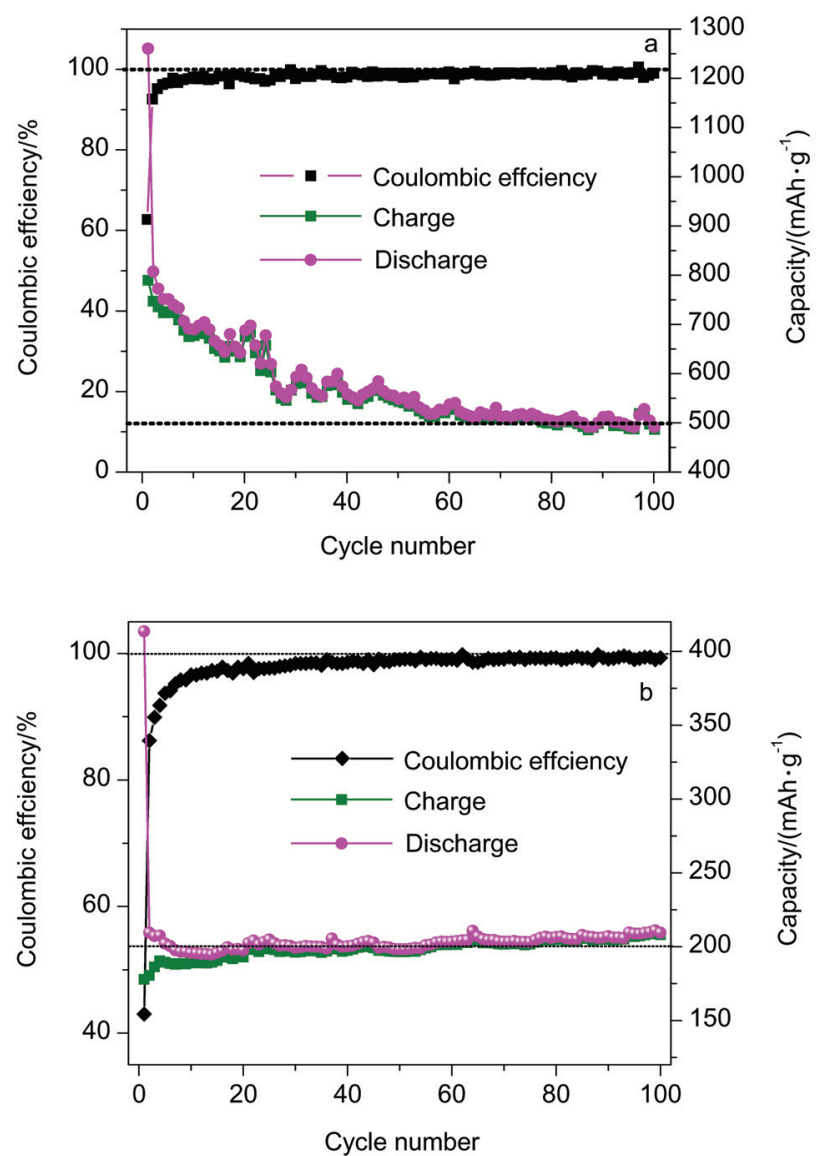

图 $7 \mathrm{CNBs}$ (a) 和 H-CNBs (b)的充放电循环曲线和库仑效率(电流密 度: $1 \mathrm{C}$; 电压范围: $0.01 \sim 3.0 \mathrm{~V}$ )

Figure 7 The cycling performance of charge/discharge and the coulombic efficiency of CNBs (a) and H-CNBs (b) between $0.01 \mathrm{~V}$ and $3.0 \mathrm{~V}$ at a current density of $1 \mathrm{C}$ 
缩紧致现象, 虽然石墨化程度与 CNBs 相比有较小幅度 的提高, 但同时表面官能团在高温环境下发生了丢失和 部分转化, 造成 H-CNBs 表面活性点的损失, 使得充放 电比容量降低.

图 8 是 $\mathrm{CNBs}$ 在电流密度为 $1 \mathrm{C}$, 电压范围是 $0.01 \sim$ $3.0 \mathrm{~V}$ 时的前三圈充放电曲线. 从充放电曲线可以看出, 第一圈循环的放电比容量很高, 可达 $1260 \mathrm{mAh} / \mathrm{g}$, 其对 应的充电比容量为 $790 \mathrm{mAh} / \mathrm{g}$, 库伦效率为 $63 \%$, 从第 二圈开始, 充放电比容量趋于平稳, 库伦效率也大大提 高, 主要是由于首次嵌入的锂离子无法可逆地全部脱出, 随着循环的进行, CNBs 与电解液得到充分接触, 充放 电容量逐渐趋于稳定, 充放电效率逐渐提高, 这也与图 $7 \mathrm{a}$ 所示的库仑效率曲线相对应. 同时, 从图中可以发现, CNBs 没有明显的充放电平台, 呈现倾斜平滑的曲线, 这主要是因为在不同的电位下发生了多个电化学过程, 可能与表面官能团、缺陷等有关.

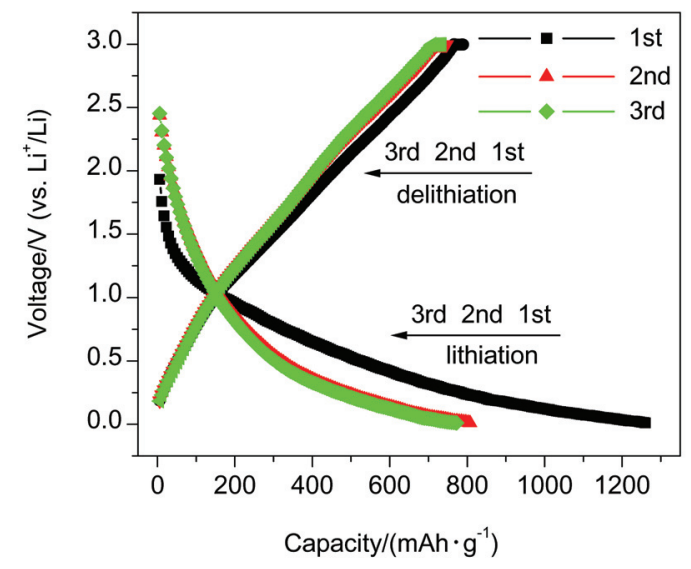

图 $8 \mathrm{CNBs}$ 的第 1, 2, 3 圈充放电曲线 (电流密度: $1 \mathrm{C}$; 电压范围: $0.01 \sim 3.0 \mathrm{~V}$ )

Figure 8 Galvanostatic charge/discharge curves of CNBs at the 1st, 2nd, and $3 \mathrm{rd}$ between $3.0 \mathrm{~V}$ and $0.01 \mathrm{~V}\left(\mathrm{vs} . \mathrm{Li}^{+} / \mathrm{Li}\right)$ at a current density of $1 \mathrm{C}$

本实验为了进一步研究 CNBs 作为负极材料的可逆 性能, 将 $\mathrm{CNBs}$ 分别在 $0.1 \mathrm{C}, 1 \mathrm{C}, 5 \mathrm{C}, 10 \mathrm{C}, 0.1 \mathrm{C}$ 下测试 其循环放电容量, 结果如图 9 所示. 由图可知, CNBs 首 次不可逆放电量可达 $1503 \mathrm{mAh} / \mathrm{g}$, 充电比容量为 745 $\mathrm{mAh} / \mathrm{g}$, 其库伦效率约为 $50 \%$, 从第二圈开始, 库伦效 率明显提高, 和图 $7 \mathrm{a}$ 所示的长循环库伦效率趋向相一 致. 经过 $0.1 \mathrm{C}, 1 \mathrm{C}, 5 \mathrm{C}$ 后, 在 $10 \mathrm{C}$ 的高电流密度下, 充 放电比容量仍可以达到 $200 \mathrm{mAh} / \mathrm{g}$, 且其库伦效率高达 98\%之上, 循环稳定性较高. 随着倍率的增大充放电比 容量在逐渐减小, 这可能因为随着充放电电流的增大, 电池内部的极化现象逐渐加重, 使得部分电极材料没有 参与到电化学反应过程中去. 倍率越大, 活性材料的利 用率越低, 材料的放电比容量也就越小. 当 CNBs 作为 负极材料经过 $0.1 \mathrm{C}, 1 \mathrm{C}, 5 \mathrm{C}, 10 \mathrm{C}$ 循环回到 $0.1 \mathrm{C}$ 下的 时候, 充放电容量又恢复到 $600 \mathrm{mAh} / \mathrm{g}$ 之上, 其库伦效 率也保持较高的稳定状态, 说明所制备的 CNBs 具有较
好的倍率性能和可逆性能，因此该材料在电化学方面有 很好的应用前景.

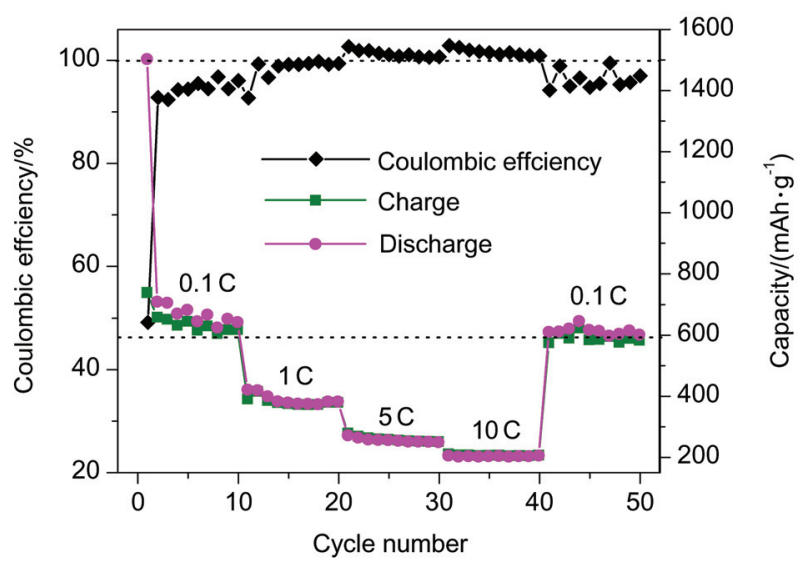

图 $9 \mathrm{CNBs}$ 的倍率充放电循环曲线和库仑效率(电压范围: $0.01 \sim 3.0$ V)

Figure 9 The rate performance of charge/discharge and the coulombic efficiency of CNBs between $0.01 \mathrm{~V}$ and $3.0 \mathrm{~V}$

\section{3 结论}

以石油沥青为碳源在空气中 $450{ }^{\circ} \mathrm{C}$ 下制备出粒径 在 $50 \sim 80 \mathrm{~nm}$ 的纳米碳球, 经 $1500{ }^{\circ} \mathrm{C}$ 氮气气氛中处理 3 $\mathrm{h}$ 后, 纳米碳球发生明显的收缩变形, 所制备的 CNBs 和 H-CNBs 石墨化程度较低, 提出了 CNBs 可能的形成 机理. 研究了所制备的 CNBs 和 H-CNBs 的电化学性质, CNBs 在 100 次循环后放电比容量仍能维持在 500 $\mathrm{mAh} / \mathrm{g}$, 库伦效率除首次较低, 其余充放电循环的库伦 效率可平稳在 $98 \%$ 左右, 同时倍率循环稳定性也比较良 好. H-CNBs 的 100 次循环充放电比容量相对较低, 但是 比较平稳, 其库伦效率在前 30 圈也不及 $\mathrm{CNBs}$ 高, 30 圈 后库伦效率呈现稳定状态. 这种 CNBs 在做锂离子二次 电池材料或者作为电极添加剂等方面将具有良好应用 前景和开发潜力.

\section{4 实验部分}

\section{1 纳米碳球的制备}

将刚玉舟、石英片分别用蒸馏水和无水乙醇超声清 洗两遍, 于鼓风干燥箱 $60{ }^{\circ} \mathrm{C}$ 烘干 $1 \mathrm{~h}$, 放于干燥器内冷 却至室温. 取约 $1 \mathrm{~g}$ 石油沥青(由克拉玛依石化提供)于 刚玉舟内, 均匀平推在舟的底部, 放于可自动鼓入洁净 空气的燃烧炉里面, 在刚玉舟上方 $5 \mathrm{~cm}$ 处放置干净的 石英片基片作为收集样品的基底, 开启炉体电源, 以 10 ${ }^{\circ} \mathrm{C} / \mathrm{min}$ 的速率升温至 $450{ }^{\circ} \mathrm{C}$, 立即关闭电源停止加热, 直至炉温自然冷却到室温，收集基底上的黑色产物，即 为 CNBs. 将 CNBs 盛放在干净的刚玉舟内, 置于直径为 $120 \mathrm{~mm}$, 长度为 $1000 \mathrm{~mm}$ 的管式炉内, 氮气流速为 20 $\mathrm{mL} / \mathrm{min}$, 以 $10{ }^{\circ} \mathrm{C} / \mathrm{min}$ 的速率升温至 $1500{ }^{\circ} \mathrm{C}$, 恒温 $3 \mathrm{~h}$, 自然冷却至室温, 收集舟内碳材料, 得 H-CNBs. 


\section{2 电池的组装}

用分析天平称量质量比为 $9: 1$ 的 CNBs 和粘结剂 聚偏二氟乙烯(PVDF), 精确至 $0.01 \mathrm{mg}$, 再将 CNBs 和 PVDF 混合均匀, 滴加适量 1-甲基-2-吡咯烷酮 $\left(\mathrm{C}_{5} \mathrm{H}_{9} \mathrm{NO}\right.$, NMP) 混合成糊状, 用刀片将糊状物均匀地涂抹在铜片 上, 置于真空干燥箱内恒温 $110{ }^{\circ} \mathrm{C}$ 保持 $12 \mathrm{~h}$, 即得到表 面平整的负极极片. 最后, 在充满氩气的手套箱中装配 成 CR2032 扣式电池, 其中手套箱内水含量与氧气含量 小于 $20 \mathrm{mg} / \mathrm{L}$. 电池内部构成依次为: 负极壳、弹片、垫 片、金属锂片、隔膜、活性物质片、正极壳. 其中锂片 为对电极, 隔膜是美国 Celgard-2499 型聚丙烯膜. 电解 液的型号为 LB-302(碳酸乙烯酯(EC)/碳酸二乙酯(DEC) $\left.1: 1(W / W) \mathrm{LiPF}_{6} 1 \mathrm{~mol} / \mathrm{L}\right)$. 装配好的电池在干燥、洁 净、透风的环境下放置一周, 以备电化学测试使用.

\section{3 纳米碳球的结构表征和电化学性能测试}

采用 X-射线粉末衍射仪(XRD)、扫描电子显微镜 (SEM)、透射电子显微镜(TEM)、拉曼光谱仪(Raman)、 红外光谱仪(IR)和热重分析仪(TGA)对样品进行形貌和 结构表征. X-射线粉末衍射仪使用的是德国 BRUKER 公司 D8 Advance; 扫描电子显微镜采用的是德国 LEO 公司的 1430VP 型; 透射电子显微镜使用的是日本日立 公司 H-600 型; 拉曼光谱仪使用的是德国 BRUKER 公 司 senterra 型; 红外光谱仪使用的是德国 BRUKER 公司 EQUINOX-55 来完成; 热重分析仪使用的是德国 NETZSCH 公司 STA449F3 型来完成. 在室温下, 采用 CT2001A 电池测试仪(武汉金诺电子有限公司)对纳米碳 球制备的电池电极在不同的电流密度下进行恒流充放 电性能测试, 电压范围为 $0.01 \sim 3.0 \mathrm{~V}$.

\section{References}

[1] Iijima, S. Nature 1991, 354, 56.

[2] Alcántara, R.; Madrigal, F. J. F.; Lavela, P.; Tirado, J. L.; Mateos, J. M. J.; Salazar, C. G.; Stoyanova, R.; Zhecheva, E. Carbon 2000, 38,
1031.

[3] Horri, B. A.; Selomulya, C.; Wang, H.-T. Int. J. Hydrogen Energy 2012, 37, 15311.

[4] Li, Y.-H.; Huang, K.-L. Acta Chim. Sinica 2011, 69, 2185. (李艳华, 黄可龙, 化学学报, 2011, 69, 2185.)

[5] Ito, Y.; Venkatesan, N.; Hirako, N.; Sugioka, N.; Takada, K. Int. J. Pharm. 2007, 337, 357

[6] Liu, Y.-C.; Qiu, X.-P.; Huang, Y.-Q.; Zhu, W.-T. Carbon 2002, 40, 2375.

[7] Uchida, M.; Aoyama, Y.; Tanabe, N.; Yanagihara, N.; Eda, N.; Ohta, A. J. Electrochem. Soc. 1995, 142, 2572.

[8] Dillon, A. C.; Jones, K. M.; Bekkedahl, T. A.; Kiang, C. H.; Bethune, D. S.; Heben, M. J. Nature 1997, 386, 377.

[9] Jiang, J.-H.; Gao, Q.-M.; Zheng, Z.-J.; Xia, K.-S.; Hu, J. Int. J. Hydrogen Energy 2010, 35, 210.

[10] Qiu, L.-B.; Sun, X.-M.; Yang, Z.-B.; Guo, W.-H.; Peng, H.-S. Acta Chim. Sinica 2012, 70, 1523. (丘龙斌, 孙雪梅, 仰志斌, 郭文瀚, 彭慧胜, 化学学报, 2012, 70, 1523.)

[11] Xia, W.; Jin, C.; Kundu, S.; Muhler, M. Carbon 2009, 47, 919.

[12] Qi, X.-S.; Zhong, W.; Yao, X.-J.; Zhang, H.; Ding, Q.; Wu, Q.; Deng, Y.; Au, C.; Du, Y. Carbon 2012, 50, 646.

[13] Mondal, K. C.; Strydom, A. M.; Tetana, Z.; Mhlanga, S. D.; Witcomb, M. J.; Havel, J.; Erasmus, R. M.; Coville, N. J. Mater. Chem. Phys. 2009, 114, 973.

[14] Yang, Y.-Z.; Liu, X.-G.; Xu, B.-S.; Li, T.-B. Carbon 2006, 44, 1661.

[15] Moothi, K.; Iyuke, S. E.; Meyyappa, M.; Falcon, R. Carbon 2012 $50,2679$.

[16] Corthals, S.; Noyen, J. V.; Geboers, J.; Vosch, T.; Liang, D.-D.; Ke, X.-X.; Hofkens, J.; Tendeloo, G. V.; Jacobs, P.; Sels, B. Carbon 2012, 50, 372.

[17] Jin, Y.-Z.; Gao, C.; Hsu, W. K.; Zhu, Y.-Q.; Huczko, A.; Bystrzejewski, M.; Roe, M.; Lee, C. Y.; Acquah, S.; Kroto, H.; Walton, D. R. M. Carbon 2005, 43, 1944.

[18] Moothi, K.; Iyuke, S. E.; Meyyappan, M.; Falcon, R. Carbon 2012, $50,2679$.

[19] Zhang, Y.-P. Ph.D. Dissertation, Dalian University of Technology, Dalian, 2004. (张云鹏, 博士论文, 大连理工大学, 大连, 2004.)

[20] Yang, Y.-Z.; Liu, X.-G.; Jia, H.-S.; Xu, B.-S. Mater. Chem. Phys. 2011, 126, 424.

[21] Liu, X.-G.; Yang, Y.-Z.; Lin, X.; Xu, B.-S.; Zhang, Y. Fuel Process. Technol. 2006, 87, 919.

[22] Yang, Y.-Z.; Liu, X.-G.; Luo, Q.-P.; Jin, L.; Xu, B.-S. New Carbon Mater. 2010, 25, 431.

[23] Li, F.-H.; Shen, Z.-M.; Xue, R.-S.; Chi, W.-D. New Carbon Mater. 2004, 19, 21. (李伏虎, 沈曾民, 薛锐生, 迟伟东, 新型碳材料, 2004, 19, 21.)

[24] Yang, Y.-Z.; Liu, X.-G.; Zhang, C.-Y.; Guo, M.-C.; Xu, B.-S. J. Phys. Chem. Solids 2010, 71, 235.

[25] Duan, L.-H.; Huang, Y.-D.; Jia, D.-Z.; Wang, X.-C.; Guo, Z.-P. Mater. Lett. 2012, 71, 151. 